\title{
Another Fallen Hash-Based RFID Authentication Protocol
}

\author{
Julio Cesar Hernandez-Castro ${ }^{1}$, Pedro Peris-Lopez ${ }^{2}$, Masoumeh Safkhani ${ }^{3}$, \\ Nasour Bagheri ${ }^{4}$, and Majid Naderi ${ }^{3}$ \\ 1 School of Computing, Portsmouth University, UK \\ Julio.Hernandez-Castro@port.ac.uk \\ 2 Computer Science Department, Carlos III University of Madrid, Spain \\ pperis@inf.uc3m.es \\ ${ }^{3}$ Electrical Eng. Department, Iran University of Science and Technology, Tehran, Iran \\ \{M_Safkhani, M_Naderi\}aiust.ac.ir \\ ${ }^{4}$ Electrical Engineering Department, Shahid Rajaee Teacher Training University, Tehran, Iran \\ NBagheri@srttu.edu
}

\begin{abstract}
In this paper, we scrutinize the security of an RFID protocol [9], which has been recently proposed, and show important vulnerabilities. Our first attack is a passive one that can disclose all secret information stored on the tags' memory. We only need to eavesdrop one session of the protocol between a tag and a legitimate reader (connected to the back-end database) and perform $O\left(2^{17}\right)$ off-line evaluations of the $P R N G$-function - while the authors wrongly claimed the complexity of any such attack would be around $2^{48}$ operations. Although the extracted information is enough to launch other relevant attacks and thus to completely rule out any of the protocol's security claims, we additionally present several attacks using alternative strategies that show the protocol is flawed in more than one way and has many exploitable weaknesses. More precisely, we present a tag impersonation attack that requires the execution of only two runs of the protocol, and has a success probability of 1 . It must be noted that this attack is, however, not applicable to the original protocol that the authors attempted to improve so, in a way, their improvement is not such. Finally, we show two approaches to trace a tag, as long as it has not updated its secret values. For all the above, we conclude that the improved protocol is even less secure than the original proposal, which is also quite insecure, and cannot be recommended.
\end{abstract}

Keywords: RFID, EPC-C1G2, Authentication, Secret Disclosure, Impersonation, Traceability.

\section{Introduction}

Radio Frequency Identification (RFID) is a wireless technology which can be employed to identify or track objects in various applications. Some common applications are animal tracking, retail, supply chain management in wholesale stores, library access control, toll payments, theft prevention, human implants, and e-passports. A typical RFID system includes a reader and a number of tags, which may range from the high

I. Askoxylakis, H.C. Pöhls, and J. Posegga (Eds.): WISTP 2012, LNCS 7322, pp. 29-37, 2012.

(c) IFIP International Federation for Information Processing 2012 
end battery-powered ones with Wi-Fi capabilities, to the low-cost that are quite constrained in resources and have no internal power, harvesting it from the readers. The tag generally includes some information related to the tag holder, and can be read/modified by the reader, which is normally securely connected to a back-end database through classical means (e.g. SSL). This technology is expected to replace barcodes in grocery and retail stores in the near future.

However, despite the multiple benefits mentioned above, security and privacy are the main concerns that slow down the rapid and widespread deployment of this technology. For instance, regarding these security concerns, only the authorized readers should be able to read or modify the information stored on the tags, only valid tags should be authenticated by a legitimate reader and it should be infeasible for a fake tag to impersonate a legitimate one. To address these multiple security and privacy requirements, several RFID mutual authentication protocols and their security analysis have already been proposed in literature, e.g. [7, 10, 11, 14]. In addition, there are several interconnected standards for RFID systems, and among them EPC global and ISO have played a major role. The Electronic Product Code Class-1 Generation-2 specification [6, 8] (EPC-C1G2 in short) was announced in 2004 by EPC Global and ratified by ISO [12]. However, later security analysis carried out on the EPC-C1 G2 specification demonstrated several security concerns [1,13]. Researchers, motivated by this, have proposed many EPC-compliant schemes -in an attempt to correct the weaknesses of the standard and improve its security- and have analyzed the security of these new schemes [2, 5, 9, 15]. Among them, one of the most recent proposals is a protocol proposed by Habibi et al. [9], which is an improvement to the Yeh et al. 's protocol [15]. Specifically, the authors analyzed the security of Yeh et al. 's protocol and proposed an improved version as a repair for the attacks they found. This new proposal is the main concern of this paper.

In this paper, we show that Habibi et al. did not succeed in their attempt, and the proposed protocol is at least as insecure as its predecessor. More precisely, they decreased the security margin of the original protocol rather than improve it, because it is possible to apply an efficient tag impersonation on the revised protocol which is not applicable to the original protocol. In addition to that, all the security problems of the original protocol remain unsolved.

Paper Organization: In $\S 2$ some preliminaries and notations are introduced. We describe the improved Yeh et al. 's protocol proposed by Habibi et al. in $\S 3$. A secret information disclosure attack is presented in $\S 4 \S 5$ and $\S[$ describe tag impersonation and traceability attacks, respectively. Finally, in $\S 7$ we extract some interestings conclusions.

\section{Preliminaries}

Throughout the paper, we use the following notation:

- $E P C_{s}$ : The 96 bits of $E P C$ code are divided into six 16-bit blocks, and then these six blocks are XORed to form $E P C_{s}$.

- DATA: The corresponding information for the tag, kept in the back-end database. 
- $K_{i}$ : The 16-bit authentication key stored in the tag to be authenticated by the backend database at the $(i+1)^{\text {th }}$ phase of authentication.

- $P_{i}$ : The 16-bit access key stored in the tag to authenticate the back-end database at the $(i+1)^{t h}$ phase of authentication.

- $K_{\text {old }}$ and $K_{\text {new }}$ : The old and new authentication keys, respectively, stored in the back-end database.

- $P_{\text {old }}$ and $P_{\text {new }}$ : The old and new access keys, respectively, stored in the back-end database.

- $C_{i}$ : The 16-bit index of the record of the $i^{\text {th }}$ tag's information in the back-end database, stored in the tag.

- $C_{\text {old }}$ and $C_{n e w}$ : The old and new back-end database indexes for the $i^{\text {th }}$ tag, respectively, stored in the back-end database .

- $X$ : The value kept as either new or old to show which key in the record of the back-end database is matched with the ones on the tag.

$-B \longleftarrow A$ : Assign the value of $A$ to $B$.

- $N_{T}$ and $N_{R}$ : 16-bit random numbers (nonces) that are generated by the tag and the reader, respectively.

- $\oplus$ : Exclusive-OR operation.

- RID: The reader identification number.

- PRNG: a 16-bit pseudo-random number generator.

- $H($.$) : A secure cryptographic hash function.$

\section{Protocol Description}

In this section we give a brief description of Habibi et al. 's protocol - see the original paper [9] for further details. This protocol has two phases: an initialization phase and an $(i+1)^{\text {th }}$ authentication phase, which are described as follows:

Initialization Phase: In this phase, the manufacturer generates random values for $K_{0}$, $P_{0}$ and $C_{0}$ respectively and sets the values of the record in the tag, i.e., $K_{i}=K_{0}$, $P_{i}=P_{0}, C_{i}=C_{0}$ and the corresponding record in the back-end database $K_{\text {old }}=$ $K_{\text {new }}=K_{0}, P_{\text {old }}=P_{\text {new }}=P_{0}, C_{\text {old }}=C_{\text {new }}=0$.

Authentication Phase: The authentication phase of Habibi et al. 's protocol, in its $(i+1)^{t h}$ run, depicted in Fig. 1. in Appendix, is as follow:

1. The reader generates a random number $N_{R}$ and sends it to the tag.

2. The tag receives $N_{R}$, generates a random number $N_{T}$, computes $M_{1}, D, E$ as shown below and finally sends $M_{1}, D, C_{i}$ and $E$ to the reader:

$M_{1} \longleftarrow P R N G\left(E P C_{s} \oplus N_{R} \oplus N_{T}\right) \oplus K_{i}$ and $D \longleftarrow N_{T} \oplus K_{i}$ and $E \longleftarrow N_{T} \oplus P R N G\left(C_{i} \oplus K_{i}\right)$.

3. Once the reader receives the message, it computes $V=H\left(R I D \oplus N_{R}\right)$ and forwards $M_{1}, D, C_{i}, E, N_{R}, V$ to the back-end database.

4. The back-end database receives $M_{1}, D, C_{i}, E, N_{R}$ and $V$. After receiving these values, it proceeds as follows:

- For each RID stored in the database (DB), it computes $H\left(R I D \oplus N_{R}\right)$ and compares it with the received $V$ to verifies the reader legitimacy. 
- If $C_{i}=0$, which means that it is the first access to the tag, it proceeds as follows, iteratively:

- Picks up an entry $\left(K_{\text {old }}, P_{\text {old }}, C_{\text {old }}, K_{\text {new }}, P_{\text {new }}, C_{\text {new }}, R I D, E P S_{s}\right.$, $D A T A)$ stored in database.

- Verifies whether $M_{1} \oplus K_{\text {old }} \stackrel{?}{=} P R N G\left(E P C_{s} \oplus N_{R} \oplus D \oplus K_{\text {old }}\right)$ or $M_{1} \oplus$ $K_{\text {new }} \stackrel{?}{=} P R N G\left(E P C_{s} \oplus N_{R} \oplus D \oplus K_{\text {new }}\right)$. If "Yes" marks $X$ as old or $n e w$ provided that the verification process is satisfied based on the new record or the old record.

- Otherwise, it uses $C_{i}$ as an index to find the corresponding record in the database and verify whether $P R N G\left(E P C_{s} \oplus N_{R} \oplus D \oplus K_{X}\right) \oplus K_{X} \stackrel{?}{=} M_{1}$. If "No" the protocol aborts.

- Verify whether $N_{T} \oplus P R N G\left(C_{X} \oplus K_{X}\right) \stackrel{?}{=} E$. If "No" the protocol aborts.

- Computes $M_{2}$ and Info as follows and forwards them to the reader: $M_{2} \longleftarrow P R N G\left(E P C_{s} \oplus N_{T}\right) \oplus P_{X}$ and Info $\longleftarrow D A T A \oplus R I D$

- If $X=n e w$, updates the database as follows: $K_{\text {old }} \longleftarrow K_{\text {new }}, K_{\text {new }} \longleftarrow \operatorname{PRNG}\left(K_{\text {new }}\right), P_{\text {old }} \longleftarrow P_{\text {new }}$, $P_{\text {new }} \longleftarrow \operatorname{PRNG}\left(P_{\text {new }}\right), C_{\text {old }} \longleftarrow C_{\text {new }}, C_{\text {new }} \longleftarrow \operatorname{PRNG}\left(N_{T} \oplus N_{R}\right)$.

- Else, $C_{\text {new }} \longleftarrow \operatorname{PRNG}\left(N_{T} \oplus N_{R}\right)$.

5. Once the reader receives the message, it extracts $D A T A$ as Info $\oplus$ RID and forwards $M_{2}$ to the tag.

6. Once the tag receives the message, it proceeds as follows:

- Verifies whether $P R N G\left(E P C_{s} \oplus N_{T}\right) \stackrel{?}{=} M_{2} \oplus P_{i}$. If "No" the protocol aborts.

- Authenticates the back-end database.

- Updates the contents kept inside as $K_{i+1} \longleftarrow P R N G\left(K_{i}\right)$, $P_{i+1} \longleftarrow \operatorname{PRNG}\left(P_{i}\right)$ and $C_{i+1} \longleftarrow \operatorname{PRNG}\left(N_{T} \oplus N_{R}\right)$.

It must be noted that the only difference between the above protocol and the original protocol, proposed by Yeh et al. [15], is that in the original protocol $M_{1}$ is computed as $M_{1}=\operatorname{PRNG}\left(E P C_{s} \oplus N_{R}\right) \oplus K_{i}$.

\section{Secret Information Disclosure Attack}

In this section we present an efficient and passive attack that retrieves any secret information in the tag, including $E P C_{s}, K_{i}$ and $P_{i}$. The main observation, which is the milestone of the given attack, is the fact that given $Y=P R N G(X)$ and the assumptions that the $P R N G$-function is a public function, and the length of $Y$ and $X$ is 16-bit, then it is possible to do an exhaustive search and find $X$ as a pre-image of $Y$ in the cost of at most $2^{16}$ off-line evaluations of $P R N G$. Following this observation, and given the fact that the tag $T_{i}$ communicates with a legitimate reader $R_{i}$, an adversary $(\mathcal{A})$ can disclose all the secret parameters of $T_{i}$ as follows:

1. Eavesdrops one session of the protocol and stores all the exchanged messages: $N_{R}, C_{i}, M_{1}=P R N G\left(E P C_{s} \oplus N_{R} \oplus N_{T}\right) \oplus K_{i}, D=N_{T} \oplus K_{i}, E=N_{T} \oplus P R N G\left(C_{i} \oplus K_{i}\right)$ and $M_{2}=P R N G\left(E P C_{s} \oplus N_{T}\right) \oplus P_{X}$. 
2. $\forall i=0, \ldots, 2^{16}-1$ does as follows:

- $K_{i} \longleftarrow i$ and $N_{T} \longleftarrow D \oplus K_{i}$

- If $E=N_{T} \oplus P R N G\left(C_{i} \oplus K_{i}\right)$ then return $K_{i}$ and $N_{T}$.

3. For the returned values of $K_{i}$ and $N_{T}$ from Step 2 and $\forall i=0, \ldots, 2^{16}-1$ does as follows:

$-E P C_{s} \longleftarrow i$,

- If $M_{1}=P R N G\left(E P C_{s} \oplus N_{R} \oplus N_{T}\right) \oplus K_{i}$ then return $E P C_{s}$.

4. For the returned values of $K_{i}$ and $N_{T}$ from Step 2 and $E P C_{s}$ from Step 3 assigns $M_{2} \oplus P R N G\left(E P C_{s} \oplus N_{T}\right)$ to $P_{i}$ and returns the following values:

$P_{\text {old }}=P_{i}, P_{\text {new }}=\operatorname{PRNG}\left(P_{i}\right), K_{\text {old }}=K_{i}, K_{\text {new }}=\operatorname{PRNG}\left(K_{i}\right), C_{\text {old }}=C_{i}$.

The complexity of the given attack is limited to eavesdropping one session of the protocol between a tag and a legitimate reader, and perform $2^{17}$ evaluations of the PRNGfunction. However, the adversary succeeds in its attack if it comes up with only one pre-image in each of Steps 2 and 3 of the given attack (it must be noted that the existence of at least one pre-image in each step is guaranteed). Otherwise, it should repeat the attack several times to come up with an unique solution. To increase the efficiency of the proposed attack, the adversary can block $M_{2}$ in the last Step of the protocol to avoid the updating of the secret values. In this case two runs of the protocol should be fairly enough to extract all given parameters.

Given all secret values of the tag, it would be easy to launch other relevant attacks with a success probability of 1 , and the cost of one execution of the protocol (e.g. traceability, tag impersonation, reader impersonation and de-synchronization).

Remark 1. It must be noted that a similar attack was applied by Habibi et al. [9] on the original protocol of Yeh et al. and the improved protocol was proposed to overcome this weaknesses. In their security analysis the authors claimed that the complexity of disclosing the secret information in their improved protocol is $2^{48}$ evaluations of the $P R N G$ function. Nevertheless, we present an efficient attack which retrieves all secret parameters with a cost of $2^{17}$ evaluations, which explicitly contradicts their claims.

Although the above attack ruins all the security properties objectives of the protocol, we continue presenting other attacks based on different strategies.

\section{Tag Impersonation Attack}

Tag impersonation attack is a forgery attack that leads to the identification of spoofed tags by a legitimate reader. In this section we show how an adversary can deceive the reader to authenticate it as a legitimate tag. In the given tag impersonation attack, the adversary, which is an active adversary, can do as follows:

Phase 1 (Learning): The adversary eavesdrops one successful run of the protocol and stores the messages exchanged between the reader and the legitimate tag including $N_{R}, M_{1}, D, C_{i}$ and $E$. 
At the end of this phase the records linked to this tag in the back-end database include $\left(K_{\text {old }}, P_{\text {old }}, C_{\text {old }}, K_{\text {new }}, P_{\text {new }}, C_{\text {new }}, R I D, E P S_{s}, D A T A\right)$ and the tag record includes $\left(K_{\text {new }}, P_{\text {new }}, C_{\text {new }}, E P S_{S}\right)$, where: $K_{\text {new }}=\operatorname{PRNG}\left(K_{\text {old }}\right), P_{\text {new }}=\operatorname{PRNG}\left(P_{\text {old }}\right)$, $C_{\text {new }}=P R N G\left(N_{T} \oplus N_{R}\right), M_{1}=P R N G\left(E P C_{s} \oplus N_{R} \oplus N_{T}\right) \oplus K_{\text {old }}, D=N_{T} \oplus K_{\text {old }}$ and $E=N_{T} \oplus P R N G\left(C_{\text {old }} \oplus K_{\text {old }}\right)$.

Phase 2 (Impersonation): To impersonate the legitimate tag, the adversary waits until the reader initiates a new protocol session, where:

1. The reader generates a random number $N_{R}^{\prime}$ and sends it to the tag.

2. After receiving $N_{R}^{\prime}$, the adversary replies with $M_{1}^{\prime}, D^{\prime}, C_{i}^{\prime}$ and $E^{\prime}$ where: $M_{1}^{\prime}=M_{1}=\operatorname{PRNG}\left(E P C_{s} \oplus N_{R}^{\prime} \oplus N_{T}\right) \oplus K_{\text {old }}, C_{i}^{\prime}=C_{\text {old }}, D^{\prime}=D \oplus N_{R} \oplus N_{R}^{\prime}=$ $N_{T} \oplus K_{i} \oplus N_{R} \oplus N_{R}^{\prime}$ and $E^{\prime}=E \oplus N_{R} \oplus N_{R}^{\prime}=N_{T} \oplus P R N G\left(C_{\text {old }} \oplus K_{\text {old }}\right) \oplus N_{R} \oplus N_{R}^{\prime}$.

3. Once the reader receives the message, it computes $V=H\left(R I D \oplus N_{R}^{\prime}\right)$ and forwards $M_{1}^{\prime}, D^{\prime}, C_{i}^{\prime}, E^{\prime}, N_{R}^{\prime}$ and $V$ to the back-end database.

4. Once the back-end database receives the message, it proceeds as follows:

- For each stored RID in the database, computes $H\left(R I D \oplus N_{R}\right)$ and compares it with the received $V$. Since the adversary has not manipulated the exchanged message from the reader to the back-end database, the back-end database authenticates the reader.

- We assume that $C_{i}^{\prime} \neq 0$, then back-end database uses $C_{i}^{\prime}=C_{i}$ as an index to find the corresponding record in the database. The record would be found in its records for the field $C_{\text {old }}$. Therefore the back-end database marks $X$ as old.

- Verifies whether $P R N G\left(E P C_{s} \oplus N_{R}^{\prime} \oplus D^{\prime} \oplus K_{\text {old }}^{\prime}\right) \oplus K_{\text {old }} \stackrel{?}{=} M_{1}^{\prime}$, where: $P R N G\left(E P C_{s} \oplus N_{R}^{\prime} \oplus D^{\prime} \oplus K_{\text {old }}\right) \oplus K_{\text {old }}=$ $P R N G\left(E P C_{s} \oplus N_{R}^{\prime} \oplus D \oplus N_{R} \oplus N_{R}^{\prime} \oplus K_{\text {old }}\right) \oplus K_{\text {old }}=$ $P R N G\left(E P C_{s} \oplus N_{R} \oplus D \oplus K_{\text {old }}\right) \oplus K_{\text {old }}=M_{1}=M_{1}^{\prime}$.

- Verifies whether $N_{T}^{\prime} \oplus P R N G\left(C_{\text {old }}^{\prime} \oplus K_{\text {old }}^{\prime}\right) \stackrel{?}{=} E^{\prime}$, where: $N_{T}^{\prime}=D^{\prime} \oplus K_{\text {old }}=N_{T} \oplus N_{R} \oplus N_{R}^{\prime} \Rightarrow N_{T}^{\prime} \oplus P R N G\left(C_{\text {old }} \oplus K_{\text {old }}\right)=$ $N_{T} \oplus N_{R} \oplus N_{R}^{\prime} \oplus P R N G\left(C_{\text {old }} \oplus K_{\text {old }}\right)=E^{\prime}$.

- Authenticates the adversary as a legitimate tag and computes $M_{2}^{\prime}$ and Info as follows, and forwards them to the reader:

$M_{2}^{\prime} \longleftarrow P R N G\left(E P C_{s} \oplus N_{T}^{\prime}\right) \oplus P_{\text {old }}^{\prime}$ and Info $\longleftarrow D A T A \oplus R I D$

- Since $X=$ old, updates the back-end database as follows: $C_{\text {new }}^{\prime} \longleftarrow \operatorname{PRNG}\left(N_{T}^{\prime} \oplus N_{R}^{\prime}\right)$.

5. Once the reader receives the message, it extracts $D A T A$ and forwards $M_{2}$ to the expected tag, which is the adversary.

Following the given attack, the adversary is authenticated by the back-end database as a legitimate tag with a probability of 1 , while the complexity of the attack is only two protocol runs with negligible time and memory requirements. It is worth to note that the given attack is not applicable to the original protocol of Yeh et al. and the complexity of the best known tag impersonation attack against the original protocol is $2^{16}$ evaluations of PRNG function [9]. It shows that Habibi et al. have decreased the security of the original protocol while trying to improve it - at least from this attack's point of view. 


\section{Traceability Attack}

In this section, we show that the improved Yeh et al. 's protocol, like the original protocol, puts at risk the location privacy of tags' holders because it is possible to track tags with a probability of 1 - between two successful runs of the authentication protocol. The following properties of the protocol are enough to trace a given tag $T_{i}$, as long as it has not updated its internal values:

1. When the reader or possibly the adversary $\mathcal{A}$, which supplants a legal reader in a mutual authentication session, sends a random number $N_{R}$ to the tag, it will answer with $M_{1}, C_{i}$, where $C_{i}$ is the tag's index in the back-end database and will remain fixed as long as the tag does not participate in another successful protocol run to update its internal values.

2. Given that the tag's reply to the reader's (or adversary) query includes $D$ and $E$, where $D=N_{T} \oplus K_{i}$ and $E=N_{T} \oplus \operatorname{PRNG}\left(C_{i} \oplus K_{i}\right)$. It can be seen that if $\mathcal{A}$ computes $Y$ as follows:

$Y \longleftarrow D \oplus E=N_{T} \oplus K_{i} \oplus N_{T} \oplus P R N G\left(C_{i} \oplus K_{i}\right)=K_{i} \oplus P R N G\left(C_{i} \oplus K_{i}\right)$

then $Y$ only depends on $K_{i}$ and $C_{i}$ and these ones will remain fixed as long as the tag does not execute a new updating phase. Hence, $Y$ can be used as a value to perfectly trace $T_{i}$.

It must be noted that this attack also works against the original protocol of Yeh et al.

\section{Conclusions}

In this paper we analyzed the security of the improved Yeh et al. 's protocol, designed to be compliant with the EPC-C1G2 standard, and being one of the most recent proposed protocols in this area. Our main attack is a passive full disclosure attack which can retrieve efficiently all the secret parameters of the tag. The cost of this attack is the eavesdropping of one protocol session and the performing of $O\left(2^{17}\right)$ off-line evaluations of the PRNG-function - while Habibi et al. claimed $O\left(2^{48}\right)$ evaluations are needed for any such attack. This attack is so powerful that it ruins all the security properties claimed by the proposed scheme. To complete this analysis, and following different strategies, we also present tag impersonation and traceability attacks that prove that these protocols are flawed in more than one way and probably do not admit an easy fixing. Summarizing, in this paper we show how the improved protocol proposed by Habibi et al. is more insecure that the one they tried to correct, which is regrettably a too common occurrence in the area.

\section{References}

1. Bailey, D.V., Juels, A.: Shoehorning Security into the EPC Tag Standard. In: De Prisco, R., Yung, M. (eds.) SCN 2006. LNCS, vol. 4116, pp. 303-320. Springer, Heidelberg (2006)

2. Burmester, M., de Medeiros, B.: The Security of EPC Gen2 Compliant RFID Protocols. In: Bellovin, S.M., Gennaro, R., Keromytis, A.D., Yung, M. (eds.) ACNS 2008. LNCS, vol. 5037, pp. 490-506. Springer, Heidelberg (2008) 
3. Burmester, M., de Medeiros, B., Munilla, J., Peinado, A.: Secure EPC Gen2 Compliant Radio Frequency Identification. In: Ruiz, P.M., Garcia-Luna-Aceves, J.J. (eds.) ADHOC-NOW 2009. LNCS, vol. 5793, pp. 227-240. Springer, Heidelberg (2009)

4. Chen, C.-L., Deng, Y.-Y.: Conformation of EPC Class-1 Generation-2 standards RFID system with mutual authentication and privacy protection. Eng. Appl. of AI 22(8), 1284-1291 (2009)

5. Chien, H.-Y., Chen, C.-H.: Mutual authentication protocol for RFID conforming to EPC Class-1 Generation-2 standards

6. Class-1 Generation-2 UHF air interface protocol standard version 1.2.0, EPCGlobal (2008), http: / /www. epcglobalinc.org/standards/

7. Duc, D.N., Kim, K.: Defending RFID authentication protocols against DoS attacks. Computer Communications 34(3), 384-390 (2011)

8. EPC Tag data standard version 1.6, EPCGlobal (2011), http: / /www. epcglobalinc.org/standards/

9. Habibi, M.H., Alagheband, M.R., Aref, M.R.: Attacks on a Lightweight Mutual Authentication Protocol under EPC C-1 G-2 Standard. In: Ardagna, C.A., Zhou, J. (eds.) WISTP 2011. LNCS, vol. 6633, pp. 254-263. Springer, Heidelberg (2011)

10. Hung-Yu, C.: SASI: A New Ultralightweight RFID Authentication Protocol Providing Strong Authentication and Strong Integrity. IEEE Transactions on Dependable and Secure Computing 4(4), 337-340 (2007)

11. Chien, H.Y.: Secure access control schemes for RFID systems with anonymity. In: Proceedings of MDM, p. 96 (2006)

12. Information technology Radio frequency identification for item management. Part 6: parameters for air interface communications at $860 \mathrm{MHz}$ to $960 \mathrm{MHz}-(2005)$,

http: / /www.iso.org

13. Peris-Lopez, P., Hernandez-Castro, J.C., Estevez-Tapiador, J.M., Ribagorda, A.: RFID specification revisited. In: The Internet of Things: From RFID to The Next-Generation Pervasive Networked Systems, pp. 6:311-6:346. Taylor \& Francis Group (2008)

14. Weis, R.-D.E.S., Sarma, S.: Security and privacy aspects of low-cost radio frequency identification systems. In: Proceedings of WiCom, pp. 2078-2080 (2007)

15. Yeh, T.-C., Wang, Y.-J., Kuo, T.-C., Wang, S.-S.: Securing RFID systems conforming to EPC Class-1 Generation-2 standard. Expert Syst. Appl. 37(12), 7678-7683 (2010) 


\section{Appendix}

\section{A Habibi et al.'s Protocol Description}

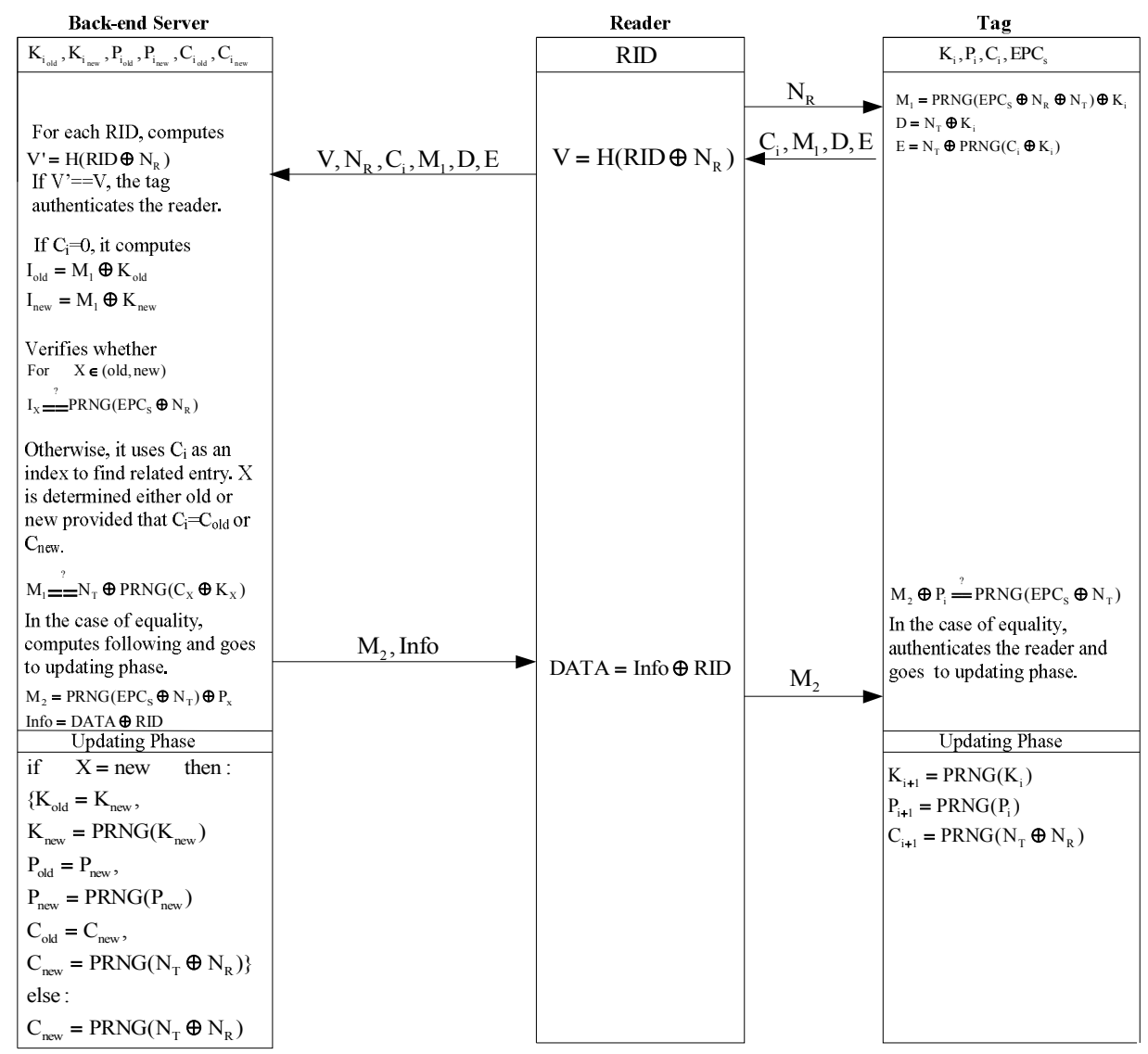

Fig. 1. Improvement of Yeh et al.'s Authentication Protocol by Habibi et al. [9] 